\title{
The National Children's Study: An Opportunity for Medical Toxicology
}

\author{
Mary Ellen Mortensen • Steven Hirschfeld
}

Published online: 23 November 2011

(C) American College of Medical Toxicology (outside the USA) 2011

\begin{abstract}
The National Children's Study (NCS) is a national longitudinal study that will prospectively investigate the influence of biological, environmental, genetic, and social factors on the health and development of US children. The NCS was mandated by the Children's Health Act of 2000 (Public Law 106-310) and is being implemented by the National Institutes of Health with input from the Centers for Disease Control and Prevention (CDC), the Environmental Protection Agency, and other federal departments and agencies. The NCS is a data-driven, evidencebased, community- and participant-informed study. Given its scale and scope, the NCS is an integrated system using several data acquisition strategies intended to provide evidenced-based design of methodologies and protocols. These strategies include the Vanguard Study, the Main Study, and formative research and sub-studies. The Vanguard Study, a pilot study, is currently underway and has been expanded from 7 to 37 study locations. The original study protocols and recruitment strategy have been field tested and revisions are
\end{abstract}

The findings and conclusions in this report are those of the authors and do not necessarily represent the views of the Centers for Disease Control and Prevention.

M. E. Mortensen $(\bowtie)$

Division of Laboratory Sciences,

National Center for Environmental Health,

Centers for Disease Control and Prevention,

4770 Buford Highway, Mailstop F-20,

Atlanta, GA 30341, USA

e-mail: zeo4@cdc.gov

\section{S. Hirschfeld}

Eunice Kennedy Shriver National Institute

of Child Health and Human Development,

31 Center Drive, Room 2A03,

Bethesda, MD 20184, USA under consideration. The CDC is collaborating with NCS in a pilot study that evaluates biological specimen protocols and will provide results on a broad array of environmental chemical exposures and nutritional indicators for a sample of Vanguard Study participants. This study is an example of the kind of collaborative opportunity that would benefit the NCS. Medical toxicologists have unique training in basic and clinical toxicology and laboratory assessments, and by partnering with study centers, both the NCS design and future NCS research projects could be enhanced.

Keywords National Children's Study · Environment · Child health · Cohort study

\section{The National Children's Study}

The National Children's Study (NCS) is intended to examine the effects of the environment on the growth, development, and health of children across the USA, following them from before birth until 21 years of age. The environment is broadly defined to include such factors as air, water, diet, family dynamics, community and cultural influences, and genetics. The longitudinal data collected and evaluated in the Study are intended to improve the health and well-being of children and contribute to understanding the role of various factors on health and disease in both childhood and adulthood. As originally conceived, the NCS was to enroll approximately 100,000 births and follow the infants to age 21 [1]. Recently, the enrollment size has undergone reevaluation. This reevaluation is based on preliminary Vanguard Study data indicating that original assumptions regarding the prevalence of pregnant women and women likely to become pregnant (determined by a screening questionnaire) were 
overly optimistic. The participation rate of pregnant women has also been lower than anticipated, as has the retention of enrollees at the end of year 1 [2]. Additional information about the NCS is available at http://www.nationalchildrensstudy. gov/Pages/default.aspx .

By developing collaborations with NCS Study Centers, medical toxicologists interested in pediatric environmental health can participate in NCS research. The NCS provides an exceptional opportunity to create a scientific learning community. With their substantial knowledge of basic and clinical toxicology, as well as laboratory assessment of drugs, chemicals, and natural toxins, medical toxicologists can offer a unique expertise and perspective to the NCS. Potential opportunities include supporting or participating in Study Center formative research projects, such as developing improved or novel methods for biological or environmental exposure assessment, or evaluating a novel biological matrix for measuring environmental analytes. Examples of recently published work by NCS investigators largely describe issues and efforts related to enrollment and retention [3-5] and exposure assessment considerations [6, 7].

\section{Background and History}

The rationale for a longitudinal cohort study was provided by the President's Task Force on Environmental Health and Safety Risks to Children, established by Executive Order 13045 in 1997 (http://nodis3.gsfc.nasa.gov/displayEO.cfm? id=EO_13045). Specifically identified was that children may be disproportionately vulnerable because of physiological and developmental differences, their behaviors, and an inability to protect themselves. The Task Force was charged with recommending strategies to identify and address children's environmental health risks and safety risks, defined as those risks attributable to products or substances that a child may be in contact with or ingest [8]. To implement the Task Force strategies, four federal workgroups were formed to focus on the pediatric outcomes of injury, asthma, cancer, and developmental disorders. The idea of a longitudinal cohort study was a product of deliberations by the Developmental Disorders Workgroup.

The NCS was authorized by the Children's Health Act of 2000 (H.R. 4365), which expanded the definition of the environment to include physical, chemical, biological, and psychosocial factors [9]. The Director of the National Institute of Child Health and Human Development (now the Eunice Kennedy Shriver National Institute of Child Health and Human Development) was directed to establish a consortium of representatives from appropriate Federal agencies to plan and implement a prospective cohort study. To provide oversight and lead study implementation, a
Program Office was established and staffed within this Institute. The other lead federal agencies are the US Environmental Protection Agency (US EPA) and Centers for Disease Control and Prevention (CDC). Within the National Institutes of Health, the National Institute of Environmental Health Sciences also has been actively involved in NCS development and planning; other institutes have provided scientific advice and expertise.

The NCS is governed by the US Congress, Office of Management and Budget, and within the Department of Health and Human Services, the National Institutes of Health Office of the Director and Eunice Kennedy Shriver National Institute of Child Health and Human Development. Federal oversight is provided by the Office of Human Research Protection and the NICHD Institutional Review Board. Advisory and oversight activities include a federal advisory committee, a steering committee of the NCS principle investigators, an independent study monitoring and oversight committee, and an interagency coordinating committee that includes US EPA, NIH, and CDC scientists.

\section{NCS Structure: A System of Research Activities}

The NCS is more accurately considered to be a set of research activities comprising an integrated system of processes and components, not just as a single study protocol. This approach allows for flexibility so activities can be reprioritized, reassigned, and reallocated when necessary. The capacity to affect rapid change within the system is required, so that, for example, a process such as recruitment or retention may be modified to achieve more desirable participation. As an integrated system, the data coming from a variety of inputs can be synthesized and analyzed, thus enhancing its scientific contribution. The data acquisition strategies highlight the scope and interdependence of the NCS, and include the following:

- Vanguard Study: A pilot study which is operational and aimed at understanding the feasibility, acceptability, and costs of recruitment, operations, and logistics, and study visits and visit assessments. The Vanguard Study will run in parallel with the Main Study, following enrolled children for 21 years. It is intended to remain dynamic in nature and content in order to evaluate multiple procedural and operational scenarios for feasibility, acceptability, and costs. Data from this study will inform the Main Study, but data will not be merged with Main Study data.

- Main Study: The study activity that will focus on exposure and outcomes assessment. Its design will be informed by the data and evidence from the Vanguard Study. 
- Sub-studies: Studies to address specific operational or scientific questions that are embedded within the Vanguard Study, and in the future, the Main Study.

- Formative Research: Research projects of a limited duration and defined scope conducted in response to technical or methodological questions that require answers in order to design the Vanguard or Main Study.

\section{National Children's Study Sample}

As originally conceived, the NCS would employ a multistage, clustered probability sample, using a national household methodology and intended to provide a representative sample of US births. The multistage sampling scheme involved selection of geographic areas, with a goal of enrolling all births to women in the sample area during the enrollment period. The considerations and challenges of this sampling design are described by Montaquila et al. [10], and the importance of a national probability sample and participant retention for the NCS to meet its intended goals is described by Ellenberg [11]. At present, alternative recruitment strategies are being considered, and the goal has been retained for the NCS to enroll a sample that will provide results that can be generalized to the US population. Retention of participants in the cohort is also critically important so the Study can maintain an unbiased sample and provide adequate numbers of children with uncommon or "rare" outcomes [11].

\section{Vanguard Study of the NCS}

The Vanguard Study began in early 2009 as a feasibility or pilot study, with seven study centers recruiting from each of their targeted the study locations. These study locations represent a diversity of population density, geographic size, and demographics for testing the recruitment, enrollment, and study visit assessment methodologies. Within these locations, efforts were made to visit all households and to determine if eligible women (pregnant or planning to become pregnant) resided in these households, and ultimately, to enroll them. The NCS Study Centers including Vanguard Center locations as well as current recruiting status and contact information are available at http://www.nationalchildrensstudy.gov/studyloca tions/pages/overview.aspx.

The study visit assessments reflected the extensive and varied exposure and outcome information to be obtained. Data collection included interview data, physical measures, biological samples, and home environmental samples prior to conception or in early pregnancy. In-person contacts with NCS participants included at-home and clinic visits during pregnancy, collection of biological samples and examination of the infant at birth, and periodic contacts with the family and child during infancy and early childhood. Table 1 provides examples of exposures and outcomes that will be taken into consideration for designing the Main Study protocols.

\section{Alternative Recruitment Sub-study}

In 2009 the NCS Program Office recognized that the single household-based methodology used for recruitment into the Vanguard Study was unlikely to yield the desired enrollment in the projected time frame and within the allocated budget. To test additional alternative methods for recruitment, additional study centers were given the opportunity to participate in a recruitment sub-study to evaluate one of three alternate recruitment strategies:

- An enhanced household-based strategy: The Study is introduced to potential participants by NCS field workers performing door-to-door enrollment in the selected communities and neighborhoods. These household visits will complement information the families receive about the Study through community engagement and media activities.

- Provider-based strategy: The Study is introduced to potential participants through their existing health care system. Women will receive information about the Study from their health care providers (including doctors, midwives, social workers, or public health nurses). These efforts will be supplemented with

Table 1 Examples of priority health exposures and outcomes in the National Children's Study

Exposure: examples

Physical: housing quality, neighborhood

Chemical: pesticides, phthalates, metals, bisphenol A, brominated flame retardants, phytoestrogens

Biological: infectious agents, endotoxins, diet

Genetics: interactions between gene and environment

Psychosocial: family structure, socioeconomic status, exposure to media and violence
Health outcome: examples
Pregnancy: preterm, birth defects

Neurodevelopment, behavior: autism, learning disabilities, conduct and behavior disorders

Injury: head trauma, injuries requiring hospitalization

Asthma: incidence and exacerbations

Obesity and physical development: obesity, diabetes, altered puberty 
general outreach activities aimed at raising awareness and recognition of the Study at a broader level.

- Two-tiered (high intensity/low intensity) strategy: Potential participants are introduced to the Study directly through public media. The catchment area for lowintensity participation is larger than the geographical study area, which allows for less restrictive Study participation and also avoids revealing the actual Study boundaries (a potential way to identify Study participants and violate confidentiality). All participants in the broader low-intensity catchment area as well as those in the smaller high-intensity catchment area will be enrolled in the low-intensity data collection effort, consisting of questionnaires offered every 6 months. After initial enrollment, participants who reside in the high-intensity areas will be recruited to join the high-intensity data collection that includes more frequent questionnaires and specimen collection, exactly as is done for the other two recruitment strategies.

Following enrollment, the protocol is identical for each study location with the exception of the broader lowintensity catchment area around the high-intensity area in the two-tiered high-low-intensity strategy. Each strategy occurs in ten locations that are geographically and demographically diverse; however, there is no attempt to have a population that can be generalized to the USA for any of the strategies. In sum, there are 30 locations engaged in the alternative recruitment sub-study. Together with the initial 7 locations that piloted household recruitment, the NCS has 37 active study locations which now comprise an expanded Vanguard Study.

The primary outcome measure for the alternative recruitment sub-study is the description of the recruitment and retention rates for the three strategies, once steady state has been reached. Steady state is defined as a study location maintaining a stable recruitment rate for three consecutive months.

The goal of the alternate recruitment sub-study is to define the effectiveness and costs of different recruitment strategies in different locations. The intent is not to select a single recruitment strategy for the NCS Main Study, but rather, to provide data for a hierarchy of approaches that may be applied in different settings.

\section{Formative Research}

The NCS has initiated numerous formative research projects. These are limited in scope and duration and are intended to augment the Vanguard Study to address specific technical questions and to provide information on the acceptability, feasibility, and costs related to research methods and process. Formative research is initiated by the NCS Program Office and funded by the NCS appropriation. The results of these projects are intended to inform the Main Study.

The formative research projects will provide data to explore new and potentially cost-effective approaches in many areas, including genetic, cognitive, and environmental assessments, which have not been evaluated from an operational perspective. Examples of formative research topic areas that are currently underway include the following:

- Real-time or near-time analysis of study samples, specimens, and measurements;

- Study logistical analysis and improvements;

- Biospecimen collection and processing;

- Environmental sample collection and processing;

- Physical measures;

- Questionnaire development and validation; and

- Study infrastructure development.

For a study on the scale and as geographically spread as the NCS, such seemingly mundane activities as blood and urine collection, handling, shipping, and storing are complex and require that detailed protocols are followed to ensure uncontaminated valid specimens. Ultimately, several million specimens may be collected and either analyzed or stored and retrieved for future analysis. Many of the challenges in biological and environmental specimen selection and collection, and the use of statistically valid subsamples for more detailed exposure assessment, have been described by others [7, 12]. Similar considerations, in addition to using the most cost-effective means for data and specimen collection, apply to each of the formative research topic areas listed above.

\section{Research Example: The NCS and CDC/NCEH Pilot Study}

The collaboration between the NCS and the National Center for Environmental Health (NCEH) Laboratory is an evaluation of the Vanguard Study protocols for biospecimen collection, handling, and storage, followed by retrieving and shipping samples of specimens from the single NCS Repository to the NCEH Laboratory. The scientific objective is to assess a broad array of environmental chemical exposures and nutritional indicators in pregnant women and their infants. The laboratory analyses will include more than 100 environmental chemicals and such nutritional biomarkers as vitamins $\mathrm{A}, \mathrm{E}$, and C, 25-hydroxy vitamin $\mathrm{D}$, and omega 3 and 6 fatty acids. The environmental chemicals are a subset of those measured in older children and adults for the National Health and Nutrition Examination Survey and reported in the Fourth National Report on Human Exposure to Environmental Chemicals 
[13]. Prenatal blood and urine, breast milk, and infant urine will be analyzed from a convenience sample of about 70 enrollees at each of seven Vanguard Centers.

From this pilot study, the NCS will obtain data on the feasibility, acceptability, and costs related to biological specimen collection. The results of the analyses will provide insight into methods for exposure information in pregnant women, new prenatal exposure information on infants, and paired maternal and infant results for selected chemicals. The results will aid in planning for the Main Study as well as future research involving NCS participants. In addition, the results can be examined relative to US population reference ranges to assess the representativeness of NCS participants.

The importance of detailed testing and evaluation of biological specimen protocols and procedures cannot be overemphasized, especially in a study involving measurement of environmental chemicals in human tissues. For the most part, environmental chemical concentrations in biological specimens are extremely low, making it critical to be aware of and to avoid external contamination sources and potential opportunities for contamination that may occur at phases of specimen collection, transport, processing, and storage. This requires close attention to ensure specimen integrity so that analytical results are valid. Table 2 presents several contamination concerns that were recognized in advance of the pilot study and how each was addressed or mitigated.

Despite careful planning to ensure biospecimen integrity, there were "lessons learned" during the pilot study that will be applied to biospecimen protocols for the Main Study. For example, during the pilot study but after cord blood collections were completed, it was determined that the samples were not suitable for analysis of environmental chemicals or nutritional biomarkers, as originally planned. The anticoagulant used in the cord blood collection was suitable for physiological analytes but not the environmental chemicals chosen for the pilot study. Subsequently, the cord blood collection procedures were modified so that future samples could be used for a broader array of analyses than would have been possible under the original protocols. Another example is that detailed specifications for specimen aliquoting at the central repository were modified when the unanticipated potential for sample crosscontamination was recognized. The preservative used in aliquoting certain specimens apparently volatilized and contaminated a nearby area where different specimens were being aliquoted.

\section{Conclusion}

The NCS will be the largest longitudinal follow-up study of US children ever undertaken. The goal of enrolling a geographically diverse sample of children across a large number of study centers creates a challenge to the goal of standardized data and specimen collection. Formative research and feasibility studies are being conducted that will inform the Main Study. CDC's NECH laboratory is collaborating with NCS to provide guidance so that biological specimens collected will be suitable for varied and reliable exposure measures. This collaboration includes a pilot study to measure a number of environmental chemicals and nutritional biomarkers in pregnant women and infants.

Medical toxicologists are uniquely trained in basic and clinical toxicology and laboratory assessment, and their collaborations with the NCS could enhance both the NCS design and future NCS research projects. Current NCS research is focused on feasibility activities such as sample collection and analysis and evaluation of novel matrices. By developing relationships with Study Centers and keeping up with the Study as it develops, medical toxicologists can explore opportunities to participate in evolving research. In the future, when the Main Study data become available, another opportunity will be available to develop research hypotheses, access stored biological or environmental specimens, and analyze publicly available data.

Table 2 Potential external contamination of biological specimens and mitigation solutions

\begin{tabular}{|c|c|c|}
\hline Chemical & Potential external contamination & Mitigation \\
\hline Metals ( $\mathrm{Pb}, \mathrm{Cd}, \mathrm{Hg}$, etc.) & $\begin{array}{l}\text { Specimen collection containers and blood } \\
\text { collection devices }\end{array}$ & Prescreen collection containers; field blanks \\
\hline Cotinine & Specimen handling by cigarette smokers & Assign nonsmokers for specimen handling \\
\hline $\begin{array}{l}\text { Brominated flame retardants } \\
\text { (PBDEs) }\end{array}$ & House dust particles & $\begin{array}{l}\text { Field blanks; maintain sealed specimen } \\
\text { containers until analysis; analyze in a dust- } \\
\text { free lab environment, i.e., biological safety } \\
\text { cabinet for specimen preparation }\end{array}$ \\
\hline $\begin{array}{l}\text { Bisphenol A, phthalate } \\
\text { metabolites, perchlorate }\end{array}$ & $\begin{array}{l}\text { Diapers or diaper inserts for infant urine } \\
\text { collection }\end{array}$ & Urine collection bag \\
\hline Triclosan & $\begin{array}{l}\text { Skin cleaning solutions or wipes used before } \\
\text { application of urine collection bag }\end{array}$ & $\begin{array}{l}\text { Wash with prepackaged distilled water and air } \\
\text { dry before application of urine collection bag }\end{array}$ \\
\hline
\end{tabular}




\section{References}

1. National Research Council (US) and Institute of Medicine (US) Panel to Review the National Children's Study Research Plan. The National Children's Study Research Plan: a review (2008) National Academies Press, Washington, DC, p. 24. Available at http://www. ncbi.nlm.nih.gov/books/NBK20656/pdf/TOC.pdf. Accessed 24 Oct 2011

2. Hirschfeld S, Songco D, Kramer BS et al (2011) National Children's Study: update in 2010. Mt Sinai J Med 78:119-125

3. Downs TJ, Ogneva-Himmelberger Y, Aupont O et al (2010) Vulnerability-based spatial sampling stratification for the National Children's Study, Worcester county, Massachusetts: capturing health-relevant environmental and sociodemographic variability. Environ Health Perspect 118:1318-1325

4. Lakes KD, Vaughan E, Jones M, Burke W, Baker D, Swanson JM (2011) Diverse perceptions of the informed consent process: implications for the recruitment and participation of diverse communities in the National Children's Study. Am J Commun Psychol. doi:10.1007/s10464-011-9450-1

5. Trasande L, Andrews HF, Goranson C et al (2011) Early experiences and predictors of recruitment success for the National Children's Study. Pediatrics 127:261-268

6. Lioy PJ, Isukapalli SS, Trasande L et al (2009) Using national and local extant data to characterize environmental exposures in the
National Children's Study: Queens county, New York. Environ Health Perspect 117:1494-1504

7. Needham LL, Ozkaynak H, Whyatt R et al (2005) Exposure assessment in the National Children's Study: introduction. Environ Health Perspect 113:1076-1082

8. The President's Task Force on Environmental Health Risks and Safety Risks to Children, Activities and Accomplishments April 14, 2003. Available at http://yosemite.epa.gov/ochp/ochpweb.nsf/ content/TaskForceSummary $1 . h t m / \$$ file/TaskForceSummary $1 . p d f$. Accessed 24 Oct 2011

9. H.R. 4365: Children's Health Act of 2000. http://www.govtrack. us/congress/bill.xpd?bill=h106-4365\&tab=summary. Accessed 24 Oct 2011

10. Montaquila JM, Brick JM, Curtin LR (2010) Statistical and practical issues in the design of a national probability sample of births for the Vanguard Study of the National Children's Study. Stat Med 29:1368-1376

11. Ellenberg JH (2010) The National Children's Study (NCS): establishment and protection of the inferential base. Stat Med 29:1360-1367

12. Ozkaynak H, Whyatt RM, Needham LL et al (2005) Exposure assessment implications for the design and implementation of the National Children's Study. Environ Health Perspect 113:1108-1115

13. Centers for Disease Control and Prevention (2009). Fourth National Report on Human Exposure to Environmental Chemicals. http:// www.cdc.gov/exposurereport/. Accessed 24 Oct 2011 